\title{
Comparison of plasma ctDNA and tissue/cytology-based techniques for the detection of EGFR mutation status in advanced NSCLC: Spanish data subset from ASSESS
}

\author{
E. Arriola ${ }^{1}$ - A. Paredes-Lario ${ }^{2} \cdot$ R. García-Gomez ${ }^{3} \cdot$ P. Diz-Tain ${ }^{4} \cdot$ M. Constenla ${ }^{5} \cdot$ C. García-Girón ${ }^{6} \cdot$ G. Márquez ${ }^{7}$. \\ M. Reck ${ }^{8} \cdot$ G. López-Vivanco ${ }^{9}$
}

Received: 20 September 2017 / Accepted: 2 March 2018 / Published online: 5 April 2018

(c) The Author(s) 2018

\begin{abstract}
Purpose The analysis of epidermal growth factor receptor (EGFR) mutations in many patients with advanced non-small-cell lung cancer (aNSCLC) has provided the opportunity for successful treatment with specific, targeted EGFR tyrosine kinase inhibitors. However, this therapeutic decision may be challenging when insufficient tumor tissue is available for EGFR mutation testing. Therefore, blood surrogate samples for EGFR mutation analysis have been suggested.

Methods Data were collected from the Spanish cohort of patients in the large, non-interventional, diagnostic ASSESS study (NCT01785888) evaluating the utility of circulating free tumor-derived DNA from plasma for EGFR mutation testing. The incidence of EGFR mutation in Spain and the level of concordance between matched tissue/cytology and plasma samples were evaluated.

Results In a cohort of 154 eligible patients, EGFR mutations were identified in 15.1 and $11.0 \%$ of tumor and plasma samples, respectively. The most commonly used EGFR mutation testing method for the tumor tissue samples was the QIAGEN Therascreen ${ }^{\circledR}$ EGFR RGQ PCR kit (52.1\%). Fragment Length Analysis + PNA LNA Clamp was used for the plasma samples. The concordance rate for $E G F R$ mutation status between the tissue/cytology and plasma samples was $88.8 \%$; the sensitivity was $45.5 \%$, and the specificity was $96.7 \%$.

Conclusions The high concordance between the different DNA sources for EGFR mutation testing supports the use of plasma samples when tumor tissue is unavailable.
\end{abstract}

Keywords Non-small-cell lung cancer $\cdot E G F R$ mutation $\cdot \operatorname{ctDNA} \cdot$ Plasma $\cdot$ Real-world $\cdot$ Liquid biopsy

\section{Introduction}

Electronic supplementary material The online version of this article (https://doi.org/10.1007/s12094-018-1855-y) contains supplementary material, which is available to authorized users.

E. Arriola

EArriola@parcdesalutmar.cat

1 Medical Oncology Department, Hospital del Mar, Passeig Marítim 25-29, 08003 Barcelona, Spain

2 Medical Oncology, Hospital Universitario Donostia, San Sebastián, Spain

3 Medical Oncology, Hospital General Universitario Gregorio Marañón, Madrid, Spain

4 Medical Oncology, Complejo Asistencial Universitario de León, León, Spain
Non-small cell lung cancer (NSCLC) represents $80-85 \%$ of all lung cancers and is a leading cause of cancer-related death worldwide [1]. It is often diagnosed at advanced

5 Medical Oncology, Complexo Hospitalario de Pontevedra, Pontevedra, Spain

6 Medical Oncology, Hospital Universitario de Burgos, Burgos, Spain

7 Medical Affairs, AstraZeneca, Madrid, Spain

8 Department of Thoracic Oncology Airway Research Center North (ARCN), German Centre for Lung Research (DZL), Grosshansdorf, Germany

9 Medical Oncology, Hospital Universitario Cruces, Baracaldo, Spain 
stages (aNSCLC). However, the management of aNSCLC, which has been traditionally treated with systemic chemotherapy, has evolved in recent years with developments in genetic profiling and the identification of driver mutations. Investigations of the activating mutations of the epidermal growth factor receptor $(E G F R)$ gene have resulted in specific therapies targeting these molecular abnormalities [2]. EGFR mutations are present in approximately $13 \%$ of European and 47\% of Japanese patients [3]. In Spanish patients, EGFR mutations have been reported in 10-16\% [4] of patients in some series and in $11.6 \%$ in a specific trial [5]. The first-generation reversible EGFR tyrosine kinase inhibitors (TKIs) gefitinib and erlotinib and the second-generation irreversible TKI afatinib elicit dramatic responses as a first-line treatment in EGFR-mutant aNSCLC patients [6-9]. Furthermore, EGFR TKIs have demonstrated superior efficacy in conjunction with chemotherapy in patients with EGFR mutation-positive aNSCLC [10-14]. However, patients will only benefit from these agents if procedures to perform $E G F R$ mutation testing are effectively implemented in a clinical setting.

A variety of methodologies are available for $E G F R$ mutation testing $[15,16]$, and direct DNA sequencing is considered the "gold standard" [17]. However, recently developed commercial real-time quantitative PCR kits have successfully increased sensitivity, reducing the amount of tumor DNA required to detect the mutation in a patient sample [15]. These molecular techniques are subject to some limitations. The primary obstacle is a lack of sufficient tumor tissue sample. Other factors include the diverse accuracy of testing techniques [18] and, logistically, the limited number of testing laboratories and poor reimbursement by healthcare systems [19]. Cytology samples have proven to be a valid source of material for mutation testing [18, 20,21]. Recently, the assessment of EGFR mutations in surrogate samples, such as serum or plasma, has gained importance [22]. In the ASSESS study [23], we observed high concordance of mutation status between matched biopsy/cytology and plasma samples, suggesting that circulating free tumor-derived DNA (ctDNA) can be isolated from the plasma or serum and could serve as a feasible sample for real-world EGFR mutation analysis. These findings were linked to robust and sensitive DNA extraction and mutation analysis methodologies. ctDNA comprises $<1 \%$ of total circulating free DNA [24]; when the previous analysis included only the subset of patients for whom DNA was re-extracted with an optimized method, the overall concordance rate and sensitivity further increased.

The present analysis from ASSESS, which originally included European and Japanese patients, aims to increase the understanding of local practices for EGFR mutation testing and the incidence of EGFR mutations in a cohort of the Spanish population under a real-world diagnostic framework.

\section{Methods}

ASSESS was a large, multicenter, non-interventional, diagnostic study (ClinicalTrials.gov NCT0178588) designed to evaluate different sample types as ctDNA sources as well as to perform $E G F R$ mutation testing in patients with aNSCLC in a real-world diagnostic setting. The study was conducted in 8 centers in Japan and 48 centers in 7 European countries, including Spain. The study protocol was approved at all participating sites. The study was conducted according to the principles of the Declaration of Helsinki and the ICH Guidelines for Good Clinical Practice and was based on the applicable regulatory requirements for non-interventional studies and AstraZeneca's policy on bioethics and human biological samples. All patients provided written informed consent. The study design has been described in full elsewhere [23] and is summarized here.

\section{Study population}

Eligible patients in European countries met the following inclusion criteria: age $\geq 18$ years; either histologically or cytologically confirmed local advanced NSCLC (stage IIIA/B [American Joint Committee on Cancer staging system]); treatment naïve and unsuitable for either curative treatment or chemoradiotherapy. Individuals with metastatic disease (stage IV) and patients with recurrent disease after previous curative treatment (resection and/or adjuvant chemotherapy) were included. DNA extraction from a tumor sample and from a fresh blood (plasma) sample for EGFR mutation testing was mandatory upon enrollment. The blood sample was collected prior to the initiation of any treatment.

\section{Study procedures}

The EGFR mutation testing methodology has been previously published [23]. In brief, centers conducted EGFR mutation testing with diagnostic tumor samples according to their local practices. All samples underwent histopathological review to ensure that they were adequate for use and were classified according to World Health Organization (WHO) guidelines [25]. In Spain, DNA extraction from blood samples and subsequent mutation testing were performed in one designated laboratory using Fragment Length Analysis + PNA Clamp technology.

The primary objective of the study was to determine the level of concordance between the EGFR mutation status obtained from tumor samples and that obtained from blood samples. The concordance rate, sensitivity, specificity, 
positive predictive value (PPV), and negative predictive value (NPV) were calculated for the tissue/cytology and plasma samples, and the exact two-sided 95\% confidence interval (CI) was obtained. The false-positive rate was defined as an EGFR mutation-positive result in plasma but mutation-negative result in tissue/cytology. The opposite definition was used for the false-negative rate. Secondary variables aimed to describe the mutation incidence and subtypes in Spain, correlations between EGFR mutation status and demographic data or disease status, and the treatment decisions following EGFR mutation testing.

\section{Statistical analyses}

The sample size calculation for this study is reported elsewhere [23]. In the analysis presented herein, only the subset of patients recruited in Spanish centers was included. EGFR mutation testing practices, EGFR mutation incidence and subtypes, and information regarding treatment choices were summarized using appropriate descriptive statistics. For the concordance rate between the EGFR mutation status in the tumor and plasma samples, we calculated the sensitivity, specificity, and the positive and negative predictive values.

\section{Results}

\section{Patients}

The ASSESS study included a total of 1311 (1288 eligible) patients, 158 (154 eligible) of whom were recruited in Spain. Four patients were excluded in this subanalysis because they did not fulfill the inclusion/exclusion criteria. The patient demographics and information regarding their NSCLC disease status are listed in Table 1; the majority were men $(74.7 \%)$, with a mean age of $64.0 \pm 10.13$ years, and $72.7 \%$ were diagnosed with adenocarcinoma.

\section{Tumor sampling and EGFR mutation testing procedures}

The biopsy samples were most commonly obtained when the patient received the current diagnosis (109/146 [74.7\%]) and from the primary tumor (109/146 [74.7\%]). The majority were collected from the lung (101/146 [69.2\%]); smaller percentages were samples collected from the lymph nodes $(12 / 146[8.2 \%])$ and pleura (9/146 [6.2\%]). The most common method for sample collection was bronchoscopy (50/146 [34.2\%]) followed by core-biopsy (30/146 [20.5\%]); a cytology sample was obtained for analysis in 27/146 $(18.5 \%)$.

Tumor tissue and cytology samples were either unavailable or insufficient for $11(7.7 \%)$ patients. Information related to the procedures for $E G F R$ mutation testing is shown in Table 2. The most commonly used EGFR mutation testing method for the tumor tissue samples was the QIAGEN therascreen ${ }^{\circledR}$ EGFR RGQ PCR kit (QIAGEN, Manchester, UK; $n=76 ; 52.1 \%$ ) followed by the Roche cobas ${ }^{\circledR}$ EGFR Mutation Test (Roche Molecular Diagnostics, Pleasanton, CA; $n=41 ; 28.1 \%)$. The EGFR mutation testing method used for all plasma samples was Fragment Length Analysis + PNA LNA Clamp. The overall mean testing turnaround times for detection were $19.2 \pm 43.69$ days with a testing success rate of $97.9 \%$ for methodologies applied to tumor samples and $35.5 \pm 20.33$ days with a mutation testing success rate of $100 \%$ for plasma samples.

\section{EGFR mutation incidence and concordance rate between sampling types}

Positive mutations of the EGFR gene were identified in 15.1 and $11.0 \%$ of the tumor and plasma samples, respectively (Table 2). When the data were categorized by tumor histology type, EGFR mutations were detected in 20/108 (18.5\%) tumor samples and $14 / 112(12.5 \%)$ plasma samples from adenocarcinoma patients and in 2/34 (5.9\%) and 3/40 (7.5\%) samples from non-adenocarcinoma individuals, respectively. The most common mutation subtypes were the single L858R substitution and exon 19 deletions in both tumor specimens (10/22 [45.5\%] and 8/22 [36.4\%], respectively) and plasma samples (4/17 [23.5\%] and 6/17 [35.3\%], respectively; Table 2).

The concordance rate between the EGFR mutation status in the tumor and plasma samples was $88.8 \%$ (95\% CI 82.5-93.5). The calculated sensitivity was $45.5 \%$ (95\% CI 24.4-67.8), with a specificity of $96.7 \%$ (95\% CI 91.8-99.1), PPV of 71.4 (95\% CI 41.9-91.6) and NPV of 90.7 (95\% CI 84.3-95.1). False-positive results were defined as EGFR mutation-positive in plasma and mutation-negative in tumor samples and were observed in 4 patients (rate of 2.8\%). Characteristics of these patients are listed in Supplementary Table S1. The corresponding false-negative rate was $8.4 \%$, corresponding to 22 patients.

\section{Treatment strategy according to EGFR mutation status}

The first-line treatment strategies according to EGFR mutation status for the population with known tumor mutation status are described in Table 3. Most patients who were EGFR mutation-positive received EGFR TKIs (19/22 [86.4\%]), including gefitinib (42.1\%), erlotinib (36.8\%) and afatinib (21\%). In those patients negative for an EGFR mutation, systemic chemotherapeutic strategies were preferred. The primary reason for treatment selection was EGFR mutation status in 79 patients (51.6\%). 
Table 1 Patient demographics and disease information of the total and tumor-evaluable Spanish populations

\begin{tabular}{|c|c|c|c|}
\hline & \multirow[t]{2}{*}{ Evaluable population } & \multicolumn{2}{|c|}{ Tumor-evaluable population } \\
\hline & & Mutated & Wild-type \\
\hline Age, mean $\pm S D$ & $64.0 \pm 10.13$ & $69.9 \pm 11.81$ & $62.7 \pm 9.58$ \\
\hline Sex (male), $n / N(\%)$ & $115 / 154(74.7)$ & $11 / 22(50.0)$ & 93/121 (76.9) \\
\hline \multicolumn{4}{|l|}{ Race, $n / N(\%)$} \\
\hline Caucasian & $154 / 154(100)$ & $22 / 22(100)$ & $121 / 121(100)$ \\
\hline Asian & $0 / 154(0.0)$ & $0 / 22(0.0)$ & $0 / 121(0.0)$ \\
\hline \multicolumn{4}{|l|}{ Smoking status } \\
\hline Non-smoker ${ }^{\mathrm{a}}, n / N(\%)$ & $27 / 154(17.5)$ & $15(68.2)$ & $12(9.9)$ \\
\hline Current smoker, $n / N(\%)$ & $53 / 154(54.4)$ & $2(9.1)$ & $49(40.5)$ \\
\hline Pack-years ${ }^{\mathrm{b}}$, mean $\pm \mathrm{SD}$ & $168.4 \pm 222.32$ & $61.2 \pm 60.99$ & $170.8 \pm 221.67$ \\
\hline $\begin{array}{l}\text { Time since first diagnosis } \\
\text { (months), mean } \pm \text { SD }\end{array}$ & $4.2 \pm 17.61$ & $2.4 \pm 6.12$ & $4.8 \pm 19.67$ \\
\hline Treatment naïve, $n / N(\%)$ & $101 / 154(65.6)$ & $14 / 22(63.6)$ & 78/121 (64.5) \\
\hline \multicolumn{4}{|l|}{ Histology, $n / N(\%)$} \\
\hline $\mathrm{ADC}$ & $112 / 154(72.7)$ & $20 / 22(90.9)$ & $88 / 121(72.7)$ \\
\hline Non-ADC & $40 / 154(26.0)$ & $2 / 22(9.1)$ & $32 / 121(26.4)$ \\
\hline \multicolumn{4}{|c|}{ WHO performance status, $n / N(\%)$} \\
\hline 0 & $38 / 154(24.7)$ & $7 / 22(31.8)$ & 28/121 (23.1) \\
\hline 1 & $88 / 154(57.1)$ & $10 / 22(45.5)$ & 71/121 (58.7) \\
\hline 2 & $25 / 154(16.2)$ & $4 / 22(18.2)$ & 20/121 (16.5) \\
\hline 3 & $3 / 154(1.9)$ & $1 / 22(4.5)$ & $2 / 121(1.7)$ \\
\hline 4 & $0 / 154(0.0)$ & $0 / 22(0.0)$ & $0 / 121(0.0)$ \\
\hline \multicolumn{4}{|c|}{ TNM (AJCC) stage status, $n / N(\%)$} \\
\hline IIIA & $9 / 154(5.8)$ & $3 / 22(13.6)$ & $4 / 121(3.3)$ \\
\hline IIIB & $9 / 154(5.8)$ & $0 / 22(0.0)$ & $7 / 121(5.8)$ \\
\hline IV & $136 / 154(88.3)$ & $19 / 22(86.4)$ & $110 / 121(90.9)$ \\
\hline \multicolumn{4}{|c|}{ TNM (AJCC) stage IV, $n / N(\%)$} \\
\hline M1a & 20/154 (13.0) & $5 / 22(22.7)$ & 15/121 (12.4) \\
\hline M1b & $51 / 154(33.1)$ & $6 / 22(27.3)$ & 41/121 (33.9) \\
\hline
\end{tabular}

$A D C$ adenocarcinoma, AJCC American Joint Committee on Cancer, SD standard deviation, TNM tumornode-metastasis, $W H O$ World Health Organization

${ }^{a}$ Non-smoker: less than 100 cigarettes in his or her lifetime

${ }^{b}$ Pack-years: (number of cigarettes smoked per day $\times$ number of years smoked)/20

\section{Discussion}

Identification of mutations in aNSCLC is essential to guide oncologists in molecular-based treatment decisions. Several clinical trials have previously suggested that plasma ctDNA detection is an appropriate surrogate marker of EGFR mutation status [26-28] In a real-world setting, the diagnostic ASSESS study showed the utility of plasmaderived ctDNA samples and good concordance based on results for 1162 matched tissue/cytology samples (89\%; sensitivity $46 \%$, specificity $97 \%$, PPV 78\%, and NPV 90\%)0 [23]. Despite this good concordance, the sensitivity in our analysis was low. Thus, the pooled sensitivity was higher in two recent meta-analyses of EGFR mutation status concordance between matched tissue/cytology and plasma samples $(62-67 \%)[29,30]$. However, it should be taken into account that sensitivity may greatly differ between a real-world study, such as the ASSESS, and clinical trials, where testing is generally well controlled and performed in a single, central laboratory, and patients are more homogenous regarding their disease stage. The present post hoc analysis of the Spanish subset of patients in the ASSESS study further confirms the utility of ctDNA analysis in plasma for the evaluation of EGFR mutation status when tumor tissue is unavailable or exhausted.

However, EGFR mutation testing practices are subject to well-described challenges. The capacity to detect mutations by ctDNA appears to be limited in some patients. We detected EGFR mutations in $15.1 \%$ of the aNSCLC patients according to tumor samples and in $11.0 \%$ according to plasma ctDNA. A lower EGFR mutation detection rate by ctDNA has been described previously [23, 31]. By 
Table 2 Evaluation of EGFR mutation testing performed using tissue/cytology and plasma samples

\begin{tabular}{lcc}
\hline & Tissue/cytology & Plasma \\
\hline Samples available, $n / N(\%)$ & $146 / 154(94.8)$ & $154 / 154(100)$ \\
Testing not performed, reasons, $n / N(\%)$ & \\
Insufficient material & $5 / 154(3.2)$ & $0 / 154(0.0)$ \\
Test not ordered/requested & $2 / 154(1.3)$ & $0 / 154(0.0)$ \\
Other & $1 / 154(0.6)$ & $0 / 154(0.0)$ \\
EGFR mutation, $n / N(\%)$ & & \\
Negative & $121 / 146(82.9)$ & $137 / 154(89.0)$ \\
Positive & $22 / 146(15.1)$ & $17 / 154(11.0)$ \\
Unknown & $3 / 146(2.1)$ & $0 / 154(0.0)$ \\
EGFR mutation subtype, $n / N(\%)$ & & \\
Exon 19 deletions & $8 / 22(36.4)$ & $6 / 17(35.3)$ \\
L858R alone & $10 / 22(45.5)$ & $4 / 17(23.5)$ \\
Exon $19+\mathrm{T} 790 \mathrm{M}$ & $0 / 22(0.0)$ & $0 / 17(0.0)$ \\
L858R + T790M & $0 / 22(0.0)$ & $1 / 17(5.9)$ \\
T790M alone & $0 / 22(0.0)$ & $3 / 17(17.6)$ \\
T790M + other & $1 / 22(4.5)$ & $1 / 17(5.9)$ \\
Other & $3 / 22(13.6)$ & $2 / 17(11.8)$ \\
\hline
\end{tabular}

${ }^{a}$ Any other mutation that occurred in combination with T790M, excluding L858R or Exon 19 deletions

contrast, in four cases, the EGFR mutation status was positive in the plasma sample and negative in the tumor sample. It is unclear if this is due to a false-negative reading in the tumor sample or false-positive reading in the ctDNA sample. Usually, false negative is found in plasma sample because of sensitivity of method and limited copy number of ctDNA. However, the heterogeneity of the mutation in the tumor sample may not be captured by biopsy analysis but may be recognized in plasma ctDNA analysis. Interestingly, 2/4 tumor samples with these discordant results were cytology blocks that may not be representative of the whole tumor genetic events and may represent heterogeneity within the tumor tissue. Moreover, the amount of DNA available from these samples may have been insufficient for detection by the PCR-based technique. Notably, 2/4 tumors harbored T790M mutations that may not be present in all tumor cells and again heterogeneity in the tumor could explain these divergent results.

In our study, discrepancies in the identification of distinct mutation subtypes were observed depending on the sample type. T790M was detected in four patients by ctDNA analysis but in only 1 patient according to the tumor tissue results. By contrast, six L858R mutations were missed in the plasma assessment, consistent with previous reports of a lower detection rate of L858R [27, 28]. A recent metaanalysis showed a wide variation in T790M detection rates, which depended on how sensitive or feasible was the technique for detection [32]. Thus, these discrepancies might reflect differences in the sensitivities of the technologies for detecting each mutation type.

Globally, in these discordant cases, clinical judgment should prevail and there are now multiple experiences of therapeutic decisions based on plasma ctDNA results where patient outcomes are concordant with expected behavior from these tumors with targeted treatments. However, whether these discordances add information to our clinical practice remains undetermined.

The use of highly sensitive detection kits is essential to avoid false-negative and false-positive results. The
Table 3 First-line treatment strategy according to EGFR mutation testing results

\begin{tabular}{lccc}
\hline & EGFR positive & EGFR negative & Total $^{\mathrm{a}}$ \\
\hline EGFR tyrosine kinase inhibitors, $n / N(\%)$ & & \\
Gefitinib & $8 / 22(40.0)$ & $0 / 121(0.0)$ & $8 / 143(5.6)$ \\
Erlotinib & $7 / 22(35.0)$ & $0 / 121(0.0)$ & $7 / 143(4.9)$ \\
Afatinib & $4 / 22(20.0)$ & $0 / 121(0.0)$ & $4 / 143(2.8)$ \\
Systemic chemotherapy, $n / N(\%)$ & & & \\
Pemetrexed & $1 / 22(5.0)$ & $60 / 121(55.6)$ & $61 / 143(42.7)$ \\
Carboplatin & $0 / 22(0.0)$ & $43 / 121(39.8)$ & $43 / 143(30.1)$ \\
Cisplatin & $1 / 22(5.0)$ & $39 / 121(36.1)$ & $40 / 143(28.0)$ \\
Vinorelbine & $1 / 22(5.0)$ & $39 / 121(36.1)$ & $40 / 143(28.0)$ \\
Paclitaxel & $0 / 22(0.0)$ & $5 / 121(4.6)$ & $5 / 143(3.5)$ \\
Gemcitabine & $0 / 22(0.0)$ & $3 / 121(2.8)$ & $3 / 143(2.1)$ \\
Docetaxel & $0 / 22(0.0)$ & $1 / 121(0.9)$ & $1 / 143(0.7)$ \\
Monoclonal antibodies, $n / N(\%)$ & & & \\
Bevacizumab & $0 / 22(0.0)$ & $3 / 121(2.8)$ & $3 / 143(2.1)$ \\
Radiotherapy, $n / N(\%)$ & $1 / 22(4.5)$ & $19 / 121(15.7)$ & $2 / 143(14.0)$ \\
Investigational agent, $n / N(\%)$ & $0 / 22(0.0)$ & $2 / 121(1.9)$ & \\
\hline
\end{tabular}

${ }^{a}$ Evaluable population with available plasma samples 
mutation-detecting Sanger sequencing method has been established as the 'gold standard' for $E G F R$ mutation testing [33], but the requirement of high-quality tumor samples and the longer turnaround time limit its utility [34, 35]. Amplification refractory mutation system (ARMS) based on PCR technology has been developed and is increasingly accepted in clinical practice. Alternatively, mutant-specific immunohistochemistry (IHC) or bead emulsion amplification and magnetics (BEAMing) has been proposed [31, 36]. Despite technical advances, variable concordance rates, sensitivity and specificity between blood-based and tumor samples for EGFR mutation detection have been reported, as shown in the global ASSESS study [23].

\section{Conclusion}

In the subset of Spanish patients in the ASSESS study, the prevalence of the EGFR mutation rate and the demographic data was as expected for a Caucasian population. The good concordance (almost 90\%) between the different DNA sources supports the use of plasma samples when tumor tissue is not available. However, this work highlights some limitations for EGFR mutation testing in the real-world setting and adds information on observed discordant results. In addition to the wide variety of testing techniques available and the differences in their sensitivity rates, this work emphasizes the importance of standardization and appropriately trained laboratories to reliably perform molecular characterization of aNSCLC patients.

Acknowledgements This work was supported by AstraZeneca and coordinated by Worldwide Clinical Trials, which also managed the database and performed the primary analyses. In collaboration with AstraZeneca, the study results were interpreted by a study steering committee. The corresponding author had full access to the study data and takes final responsibility for the decision to submit for publication.

Funding This study was funded by AstraZeneca.

\section{Compliance with ethical standards}

Conflict of interest Dr. Arriola has received personal fees from Boehringer Ingelheim, BMS, and Eli Lilly; travel expenses from Astra Zeneca and Boehringer Ingelheim. Dr. Diz-Tain has received research grants to institution from Astra-Zeneca, research grants from AstraZeneca, advisory honorarium from Boehringer Ingelheim, BMS and Eli Lilly, and travel grants from Bristol Myers Squibb, Pfizer, Roche and Merck and Co. Dr. Marquez is full-time employee of AstraZeneca. Dr. Reck has received honoraria for lectures and consultancy from AstraZeneca, Roche/Genentech, BMS, MSD, Merck, Lilly, BoehringerIngelheim, Pfizer, Novartis, Celgene. Dr. Paredes-Lario, Dr. GarcíaGómez, Dr. Constenla, Dr. García-Girón and Dr. López-Vivanco declare that they have no conflict of interest.

Research involving human participants and/or animals This study has been approved by the appropriate ethics committee and have therefore been performed in accordance with the ethical standards laid down in the 1964 Declaration of Helsinki and its later amendments.

Informed consent All individual participants gave their informed consent prior to their inclusion in the study.

Open Access This article is distributed under the terms of the Creative Commons Attribution 4.0 International License (http://creativeco mmons.org/licenses/by/4.0/), which permits unrestricted use, distribution, and reproduction in any medium, provided you give appropriate credit to the original author(s) and the source, provide a link to the Creative Commons license, and indicate if changes were made.

\section{References}

1. National Cancer Institute. SEER cancer statistics review, 19752012. http://seer.cancer.gov/csr/1975_2012/. Accessed 6 Jul 2016.

2. Mok TS, Wu YL, Thongprasert S, Yang CH, Chu DT, Saijo N, et al. Gefitinib or carboplatin-paclitaxel in pulmonary adenocarcinoma. N Engl J Med. 2009;361:947-57.

3. Dearden S, Stevens J, Wu YL, Blowers D. Mutation incidence and coincidende in NSCLC: meta-analyses by ethnicity and histology (mutMap). Ann Oncol. 2013;24:2371-6.

4. García-Campelo R, Bernabé R, Cobo M, Corral J, Coves J, Dómine $\mathrm{M}$, et al. SEOM clinical guidelines for the treatment of non-small cell lung cancer (NSCLC) 2015. Clin Transl Oncol. 2015;17:1020-9.

5. Esteban E, Majem M, Martinez Aguillo M, Martinez Banaclocha N, Dómine M, Gómez Aldaravi L, et al. Prevalence of EGFR mutations in newly diagnosed locally advanced or metastatic nonsmall cell lung cancer Spanish patients and its association with histological subtypes and clinical features: the Spanish REASON study. Cancer Epidemiol. 2015;39:291-7.

6. Lynch TJ, Bell DW, Sordella R, Gurubhagavatula S, Okimoto RA, Brannigan BW, et al. Activating mutations in the epidermal growth factor receptor underlying responsiveness of nonsmall-cell lung cancer to gefitinib. N Engl J Med. 2004;350:2129-39.

7. Paez JG, Jänne PA, Lee JC, Tracy S, Greulich H, Gabriel S, et al. EGFR mutations in lung cancer: correlation with clinical response to gefitinib therapy. Science. 2004;304:1497-500.

8. Pao W, Miller V, Zakowski M, Doherty J, Politi K, Sarkaria I, et al. EGF receptor gene mutations are common in lung cancers from "never smokers" and are associated with sensitivity of tumors to gefitinib and erlotinib. Proc Natl Acad Sci USA. 2004;101:13306-11.

9. Kato T, Yoshioka H, Okamoto I, Yokoyama A, Hida T, Seto $\mathrm{T}$, et al. Afatinib versus cisplatin plus pemetrexed in Japanese patients with advanced non-small cell lung cancer harboring activating EGFR mutations: subgroup analysis of LUX-Lung 3. Cancer Sci. 2015;106:1202-11.

10. Douillard JY, Ostoros G, Cobo M, Ciuleanu T, Cole R, McWalter $\mathrm{G}$, et al. Gefitinib treatment in EGFR mutated caucasian NSCLC: circulating-free tumor DNA as a surrogate for determination of EGFR status. J Thorac Oncol. 2014;9:1345-53.

11. Mitsudomi T, Morita S, Yatabe Y, Negoro S, Okamoto I, Tsurutani J, et al. Gefitinib versus cisplatin plus docetaxel in patients with non-small-cell lung cancer harbouring mutations of the epidermal growth factor receptor (WJTOG3405): an open label, randomised phase 3 trial. Lancet Oncol. 2010;11:121-8.

12. Mok TS, Wu YL, Thongprasert S, Yang CH, Chu DT, Saijo N, et al. Gefitinib or carboplatin-paclitaxel in pulmonary adenocarcinoma. N Engl J Med. 2009;361:947-57. 
13. Rosell R, Carcereny E, Gervais R, Vergnenegre A, Massuti B, Felip E, et al. Erlotinib versus standard chemotherapy as firstline treatment for European patients with advanced EGFR mutation-positive non-small-cell lung cancer (EURTAC): a multicentre, open-label, randomised phase 3 trial. Lancet Oncol. 2012;13:239-46.

14. Zhou C, Wu YL, Chen G, Feng J, Liu XQ, Wang C, et al. Erlotinib versus chemotherapy as first-line treatment for patients with advanced EGFR mutation-positive non-small-cell lung cancer (OPTIMAL, CTONG-0802): a multicentre, open-label, randomised, phase 3 study. Lancet Oncol. 2011;12:735-42.

15. Angulo B, Conde E, Suárez-Gauthier A, Plaza C, Martínez R, Redondo $\mathrm{P}$, et al. A comparison of EGFR mutation testing methods in lung carcinoma: direct sequencing, real-time PCR and immunohistochemistry. PLoS One. 2012;7:e43842.

16. Goto K, Satouchi M, Ishii G, Nishio K, Hagiwara K, Mitsudomi $\mathrm{T}$, et al. An evaluation study of EGFR mutation tests utilized for non-small-cell lung cancer in the diagnostic setting. Ann Oncol. 2012;23:2914-9.

17. Bakker E. Is the DNA sequence the gold standard in genetic testing? Quality of molecular genetic tests assessed. Clin Chem. 2006;52:557-8.

18. Ellison G, Zhu G, Moulis A, Dearden S, Speake G, McCormack R. EGFR mutation testing in lung cancer: a review of available methods and their use for analysis of tumour tissue and cytology samples. J Clin Pathol. 2013;66:79-89.

19. Ellis PM, Verma S, Sehdev S, Younus J, Leighl NB. Challenges to implementation of an epidermal growth factor receptor testing strategy for non-small-cell lung cancer in a publicly funded health care system. J Thorac Oncol. 2013;8:1136-41.

20. da Cunha Santos G, Saieg MA, Geddie W, Leighl N. EGFR gene status in cytological samples of nonsmall cell lung carcinoma: controversies and opportunities. Cancer Cytopathol. 2011;119:80-91.

21. Hagiwara K, Kobayashi K. Importance of the cytological samples for the epidermal growth factor receptor gene mutation test for non-small cell lung cancer. Cancer Sci. 2013;104:291-7.

22. Yung TK, Chan KC, Mok TS, Tong J, To KF, Lo YM. Singlemolecule detection of epidermal growth factor receptor mutations in plasma by microfluidics digital PCR in non-small cell lung cancer patients. Clin Cancer Res. 2009;15:2076-84.

23. Reck M, Hagiwara K, Han B, Tjulandin S, Grohé C, Yokoi T, et al. ctDNA determination of EGFR mutation status in european and japanese patients with advanced NSCLC: the ASSESS study. J Thorac Oncol. 2016;11:1682-9.

24. Diaz LA Jr, Bardelli A. Liquid biopsies: genotyping circulating tumor DNA. J Clin Oncol. 2014;32:579-86.
25. Brambilla E, Travis WD, Colby TV, Corrin B, Shimosato Y. The new World Health Organization classification of lung tumours. Eur Respir J. 2001;18:1059-68.

26. Douillard JY, Ostoros G, Cobo M, Ciuleanu T, Cole R, McWalter $\mathrm{G}$, et al. Gefitinib treatment in EGFR mutated caucasian NSCLC: circulating-free tumor DNA as a surrogate for determination of EGFR status. J Thorac Oncol. 2014;9:1345-53.

27. Mok T, Wu YL, Lee JS, Yu CJ, Sriuranpong V, Sandoval-Tan J, et al. Detection and dynamic changes of EGFR mutations from circulating tumor DNA as a predictor of survival outcomes in NSCLC patients treated with first-line intercalated erlotinib and chemotherapy. Clin Cancer Res. 2015;21:3196-203.

28. Karachaliou N, Mayo-de las Casas C, C Queralt, de Aguirre I, Melloni B, F Cardenal, et al. Association of EGFR L858R mutation in circulating free DNA with survival in the EURTAC trial. JAMA Oncol. 2015;1:149-57.

29. Qiu M, Wang J, Xu Y, Ding X, Li M, Jiang F, et al. Circulating tumor DNA is effective for the detection of EGFR mutation in non-small cell lung cancer: a meta-analysis. Cancer Epidemiol Biomark Prev. 2015;24:206-12.

30. Luo J, Shen L, Zheng D. Diagnostic value of circulating free DNA for the detection of EGFR mutation status in NSCLC: a systematic review and meta-analysis. Sci Rep. 2014;4:6269.

31. Douillard JY, Ostoros G, Cobo M, Ciuleanu T, Cole R, McWalter $\mathrm{G}$, et al. Gefitinib treatment in EGFR mutated caucasian NSCLC: circulating-free tumor DNA as a surrogate for determination of EGFR status. J Thorac Oncol. 2014;9:1345-53.

32. Chen LY, Molina-Vila MA, Ruan SY, Su KY, Liao WY, Yu KL, et al. Coexistence of EGFR T790M mutation and common activating mutations in pretreatment non-small cell lung cancer: a systematic review and meta-analysis. Lung Cancer. 2016;94:46-53.

33. Bakker E. Is the DNA sequence the gold standard in genetic testing? Quality of molecular genetic tests assessed. Clin Chem. 2006;52:557-8.

34. Ogino S, Kawasaki T, Brahmandam M, Yan L, Cantor M, Namgyal C, et al. Sensitive sequencing method for KRAS mutation detection by pyrosequencing. J Mol Diagn. 2005;7:413-21.

35. Li J, Wang L, Mamon H, Kulke MH, Berbeco R, Makrigiorgos GM. Replacing PCR with COLD-PCR enriches variant DNA sequences and redefines the sensitivity of genetic testing. Nat Med. 2008;14:579-84.

36. Liu Y, Liu B, Li XY, Li JJ, Qin HF, Tang CH, et al. A comparison of ARMS and direct sequencing for EGFR mutation analysis and tyrosine kinase inhibitors treatment prediction in body fluid samples of non-small-cell lung cancer patients. J Exp Clin Cancer Res. 2011;30:111. 